\title{
Impact of the unit cell choice on the efficiency of dispersion curve calculations using GBMS
}

\author{
Vanessa Cool* \\ DMMS lab, Flanders Make \\ Division LMSD, Department of Mech. \\ Engineering, KU Leuven \\ Celestijnenlaan 300 - box 2420 \\ Heverlee B-3001, Belgium \\ e-mail: vanessa.cool@kuleuven.be
}

\author{
Lucas Van Belle \\ DMMS lab, Flanders Make \\ Division LMSD, Department of Mech. \\ Engineering, KU Leuven \\ Celestijnenlaan 300 - box 2420 \\ Heverlee B-3001, Belgium \\ e-mail: lucas.vanbelle@kuleuven.be
}

\author{
Claus Claeys \\ DMMS lab, Flanders Make \\ Division LMSD, Department of Mech. \\ Engineering, KU Leuven \\ Celestijnenlaan 300 - box 2420 \\ Heverlee B-3001, Belgium \\ e-mail: claus.claeys@kuleuven.be \\ Wim Desmet \\ DMMS lab, Flanders Make \\ Division LMSD, Department of Mech. \\ Engineering, KU Leuven \\ Celestijnenlaan 300 - box 2420 \\ Heverlee B-3001, Belgium \\ e-mail: wim.desmet@kuleuven.be
}

Elke Deckers

DMMS lab, Flanders Make

Engineering, KU Leuven, Campus Diepenbeek

Wetenschapspark 27

Diepenbeek B-3590, Belgium

e-mail: elke.deckers@kuleuven.be

\begin{abstract}
Recently, the potential of metamaterials and phononic crystals to cope with conflicting requirements of obtaining lightweight structures with desirable noise and vibration properties has been demonstrated. These, often periodic, structures are commonly studied based on their representative unit cell of which the vibro-acoustic performance is examined by means of their wave propagation, visualized by dispersion curves. Typically, the unit cell is discretized using a finite element technique to capture the possibly complex geometry. This leads to a high computation cost for the dispersion curve calculation which can be strongly reduced by applying modal based model order reduction techniques such as the (generalized) Bloch mode synthesis. In this paper, the choice of the unit cell is shown to have an impact on the dispersion curve calculation time. Moreover, the efficiency of (generalized) Bloch mode synthesis strongly depends on the unit cell choice. The highest reduction in computation time is accomplished when the number of boundary degrees-offreedom is limited.
\end{abstract}

\footnotetext{
${ }^{*}$ Corresponding author.
}

\section{Introduction}

Over the years, there has been an extensive scientific and technical interest in exploiting, often periodic, structures to control waves in different disciplines, e.g. optical, electromagnetic, acoustic waves etc. [1]. In light of noise, vibration and harshness (NVH) performances, one is interested in obtaining noise and vibration attenuation in targeted frequency ranges. Recently, metamaterials and phononic crystals have come to the fore to cope with the conflicting requirements of designing lightweight structures with desirable $\mathrm{NVH}$ performance. These structures are engineered geometries out of conventional materials which exhibit unconventional properties, i.e. stop bands in specific frequency ranges where wave transmission is suppressed. In the past decades, research is primarily executed towards $1 \mathrm{D}$ and $2 \mathrm{D}$ periodic plane structures which are most common constructed out of a beam or plate host structure with periodically added masses or resonators $[2,3]$. In recent literature, full 3D periodic metamaterials are encountered which are able to render omnidirectional stop bands due to the periodicity in all three directions $[4,5]$. 
The performance of these periodic structures is often analyzed using dispersion curves which contain information on the wave propagation of the corresponding infinite periodic structure [1]. To this end, the periodicity of the structure is exploited and the input for the dispersion curve calculation is the representative unit cell (UC) of the structure. The three most common techniques to discretize the UC are the finite difference method, the plane-wave expansion method and the finite element (FE) technique [6]. The FE technique has as advantages its suitability to discretize complex designs and is readily available in commercial FE software packages. The dispersion eigenvalue problem is constructed by applying Bloch-Floquet periodicity boundary conditions (BCs) [7] to the boundary degrees-of-freedom (DOFs) of the UC. Several approaches exist to determine the dispersion curves, mostly denoted by the direct and indirect technique $[1,8]$. Due to the complex dynamics related to the often sub-wavelength sized UCs, a sufficiently refined FE model is required leading to a significant amount of DOFs. The computational cost to determine the dispersion curves as such increases quickly with the complexity of the UCs, i.e. number of DOFs, and the required resolution which one wants to obtain, i.e. the number of times the eigenvalue problem needs to be solved. These phenomena are magnified when going towards $3 \mathrm{D}$ periodic structures which inherently contain more DOFs.

To overcome this high computational load to compute the full order model (FOM), model order reduction (MOR) techniques have come to the fore. Many MOR techniques are already applied for the analysis of large dynamical systems, e.g. [9-12]. On UC level, these techniques aim to reduce the UC system matrices by constructing a reduced model with less DOFs while maintaining the relevant dynamic information included in the UC model. Many MOR techniques for periodic structures start by dividing the system in its interior and boundary part. Commonly, the MOR techniques first focus on the interior part, after which the boundary part is envisaged. This can become of importance as many 3D UCs encountered in recent literature contain a relatively large amount of boundary DOFs in comparison with the amount of interior DOFs, e.g. [4,5, 13]. Different methodologies can be followed to set up a reduced system. A first class of techniques is based on the condensation of the interior DOFs of the UC, i.e. static condensation (or Guyan reduction) and dynamic condensation [6]. A second class is denoted as projection-based modal reduction techniques which can be further divided into wave-based techniques, approaches following the component mode synthesis and hybrid methods. The reduced Bloch mode expansion (RBME) technique is the best known wave-based MOR [14]. The reduction basis in this technique is constructed based on computed wave modes at high symmetry points in the wave domain. Several hybrid methods, e.g. $[15,16]$, exist in literature which combine the wave-based approach and component mode synthesis. The most commonly applied MOR techniques based on the component mode synthesis are the Bloch mode synthesis (BMS) and generalized BMS (GBMS), both proposed by Krattiger et al. [6,17]. In the BMS method, the number of interior DOFs of the UC is reduced using a truncated set of normal modes while the number of boundary DOFs is preserved. Later on, this technique was extended to the GBMS method to also reduce the boundary DOFs by including a set of boundary modes in the reduction basis. In [18], different interface reduction strategies are compared. In this work, it is opted to work with the $(\mathrm{G}) \mathrm{BMS}$ technique for two reasons. (i) Compared to the RBME and hybrid methods, the (G)BMS allows to reduce the UC before solving the dispersion eigenvalue problem, making the reduced model independent of the propagation constant. (ii) Moreover, the (G)BMS techniques have been shown to strongly reduce the dispersion curve calculation time of $2 \mathrm{D}$ periodic metamaterials with a large amount of DOFs ( $>15000 \mathrm{DOFs}$ ) [19]. Although the $(\mathrm{G}) \mathrm{BMS}$ techniques are widely applied to $1 \mathrm{D}$ and $2 \mathrm{D}$ periodic structures, their efficiency to reduce $3 \mathrm{D}$ periodic structures has yet to be shown. Going towards 3D structures comes at an increased cost not only due to the increased number of wave propagation direction of interest, but most often also due to an increased complexity of the UC leading to more DOFs. Moreover, the impact on the efficiency of the (G)BMS when applied to the, often encountered, 3D UCs with a large number of boundary DOFs, is unknown.

In this work, the $(\mathrm{G}) \mathrm{BMS}$ technique is applied to a $3 \mathrm{D}$ periodic phononic crystal containing a large amount of DOFs ( $>15000 \mathrm{DOFs}$ ). Via a numerical case study, a comparison is executed between the dispersion curve calculation of two UCs representing the same periodic structure, but which differ in their ratio of interior to boundary DOFs. For completeness, the comparison is done for both the FOM UCs as when the (G)BMS techniques are applied. As such, the impact of the UC choice on the efficiency of the $(G) B M S$ and the attainable reduction in computation time with these techniques is investigated.

This paper is structured as follows. In Section 2, the dispersion curve calculation and the (G)BMS technique are described. Section 3 details the considered unit cells and the corresponding numerical model choices. Next, Section 4 compares the FOM and reduced order model (ROM) dispersion curve calculations for the different considered UC definitions. The final section summarizes the main conclusions.

\section{Methodology}

This section briefly describes the finite element based dispersion curve calculation using the Wave and Finite Element Method (WFEM) as well as the BMS and GBMS model reduction techniques.

\subsection{Dispersion curve calculation}

Dispersion curves describe the wave propagation through a structure by solving the relation between the frequency $\omega$ and wave propagation constant $\mu$, i.e. $\left(\mu_{x}, \mu_{y}, \mu_{z}\right)$ in the $3 \mathrm{D}$ periodic case. The input for the dispersion curve calculation is the representative UC of the structure, often descritized by a FE method. The dispersion relation is derived starting from the equations of motion of the undamped FE UC without external excitation:

$$
\left(\mathbf{K}-\omega^{2} \mathbf{M}\right) \mathbf{q}=\mathbf{0}
$$


with $\mathbf{q}$ the general nodal displacement and $\mathbf{M}, \mathbf{K}$ the mass and stiffness matrices. The Bloch-Floquet theorem [7], which governs the periodic displacement and forcing conditions in infinite periodic structures, is applied to the UC. Practically, the Bloch-Floquet BCs prescribe a relation between the compatible boundary DOFs (e.g. left and right set) and are applied to the structure using the periodicity matrix $\Lambda$ [8]: $\mathbf{q}=\boldsymbol{\Lambda} \tilde{\mathbf{q}}$ with $\tilde{\mathbf{q}}$ the periodic DOF vector. Rewriting Eq. (1) using the periodicity conditions leads to the dispersion eigenvalue problem:

$$
\left(\tilde{\mathbf{K}}-\omega^{2} \tilde{\mathbf{M}}\right) \tilde{\mathbf{q}}=\mathbf{0}
$$

with

$$
\tilde{\mathbf{K}}=\mathbf{\Lambda}^{H} \mathbf{K} \boldsymbol{\Lambda}, \quad \tilde{\mathbf{M}}=\mathbf{\Lambda}^{H} \mathbf{M} \boldsymbol{\Lambda},
$$

with $H$ the Hermetian transpose of the matrix. Several approaches exist to solve Eq. (2) [1,8]. In this work, the inverse or $\omega(\boldsymbol{\mu})$-approach is adopted in which a set of real propagation constants $\left(\mu_{x}, \mu_{y}, \mu_{z}\right)$ are imposed to the problem and the corresponding frequencies are determined. By exploiting the symmetry of the $\mathrm{UC}$, the $\left(\mu_{x}, \mu_{y}, \mu_{z}\right)$-sets are selected only along the Irreducible Brillouin Contour (IBC) of the UC [20]. The dispersion eigenvalue problem, i.e. Eq. (2), is performed using the built-in function eigs of Matlab.

\subsection{Bloch mode synthesis}

The BMS, proposed by Krattiger et al. [17], is a projection-based MOR technique which reduces the number of interior UC DOFs. In order to set up the reduction base, the DOFs of the UC (and system matrices) are divided into interior $(I)$ and boundary $(A)$ DOFs. Next, a projection matrix $\mathbf{B}$ is constructed and applied to the full order system matrices $(\mathbf{M}, \mathbf{K})$ to set up the approximate (and reduced) system matrices $(\mathcal{M}, \mathcal{K})$ :

$$
\mathcal{M}=\mathbf{B}^{T} \mathbf{M B}, \quad \mathcal{K}=\mathbf{B}^{T} \mathbf{K B},
$$

in which $T$ denotes the transpose of the matrix. The reduction base is set up using a Craig-Bampton approach, in which the interior DOFs are represented by fixed interface normal modes $\boldsymbol{\Phi}_{I}$ and the influence of the boundary motion on the interior is given by a set of static constraint modes $\Psi_{I A}$ :

$$
\begin{aligned}
& \mathbf{B}=\left[\begin{array}{cc}
\boldsymbol{\Phi}_{I} & \boldsymbol{\Psi}_{I A} \\
\mathbf{0} & \mathbf{I}
\end{array}\right] ; \\
& \left(\mathbf{K}_{I I}-\omega_{i}^{2} \mathbf{M}_{I I}\right) \phi_{I}^{i}=\mathbf{0} \quad \text { and } \quad \mathbf{\Psi}_{I A}=-\mathbf{K}_{I I}^{-1} \mathbf{K}_{I A} \text {, }
\end{aligned}
$$

in which $\boldsymbol{\Phi}_{I}=\left[\begin{array}{llll}\phi_{I}^{1} & \phi_{I}^{2} & \ldots & \phi_{I}^{n_{I}}\end{array}\right]$ and $n_{I}$ the number of modes taken into account. The number of included fixed interface modes $n_{I}$ is selected using a frequency-based mode selection: the modes up to twice the maximum frequency of interest are included in the modal basis [17]. Due to the static constraint modes, the sparsity of the full order UC matrices is not preserved. This potentially has a large impact on the computation time, which is expected to become outspoken when a large number of boundary DOFs is present. After the reduction, the Bloch-Floquet $\mathrm{BCs}$ are applied to the reduced $\mathrm{UC}$ and the dispersion curves are calculated as described in previous section.

\subsection{Generalized Bloch mode synthesis}

Krattiger et al. [6] proposed the GBMS which builds further upon the BMS technique. The BMS only reduces the interior DOFs, resulting in possibly large reduced models for UCs with a lot of boundary DOFs. Therefore, the GBMS additionally performs a local interface modal reduction to reduce these number of boundary DOFs. The boundary reduction uses a set of normal modes calculated from the reduced submatrices $\mathcal{M}_{A A}$ and $\mathcal{K}_{A A}$, cf. Eq. (4):

$$
\left(\mathcal{K}_{A A}-\omega_{j}^{2} \mathcal{M}_{A A}\right) \phi_{A}^{j}=\mathbf{0}
$$

with

$$
\boldsymbol{\Phi}_{A}=\left[\begin{array}{llll}
\phi_{A}^{1} & \phi_{A}^{2} & \ldots & \phi_{A}^{n_{A}}
\end{array}\right]
$$

in which $n_{A}$ represents the number of boundary modes taken into account. It is recommended to include a minimal number of boundary modes $n_{A}$ instead of a frequency-dependent threshold used to select $n_{I}$ in the BMS [21]. Therefore, the number $n_{A}$ is chosen with trial and error such that the GBMS still give backs a reduction while preserving a good accuracy. Because the reduction is applied before imposing the BlochFloquet BCs, the reduced boundary DOFs need to express the same compatibility conditions as the FOM (Eq. (3)). To include these conditions, the modeshapes $\boldsymbol{\Phi}_{A}$ are partitioned according to the boundary parts, e.g. $\boldsymbol{\Phi}_{L}$ for the left boundary DOFs. Next, a single modal basis per required compatible boundary DOFs set is constructed by concatenating the corresponding partial bases, e.g. for the left and right boundary: $\boldsymbol{\Phi}_{L R}=\left[\boldsymbol{\Phi}_{L}, \boldsymbol{\Phi}_{R}\right]$. To avoid singularity issues, a singular value decomposition is used to orthogonalize the basis per set. In this way, a new projection matrix per set of boundaries is obtained, which are gathered in the total projection matrix $\mathbf{L}$ for all boundaries [6]. Finally, the projection matrix $\mathbf{B}$ of the BMS is extended to include the boundary reduction:

$$
\mathbf{B}=\left[\begin{array}{cc}
\mathbf{\Phi}_{I} & \mathbf{\Psi}_{I A} \mathbf{L} \\
\mathbf{0} & \mathbf{L}
\end{array}\right]
$$

The UC system matrices are reduced by executing the projection given in Eq. (4). In this work, the GBMS technique is used without including a residual interior mode correction [6]. After the GBMS is executed, the Bloch-Floquet BCs are applied to the reduced UC system matrices and the dispersion curves can be calculated. The eigenvalue problems, i.e. Eqs. (5) and (6), are again performed using the built-in function eigs of Matlab. 
a)

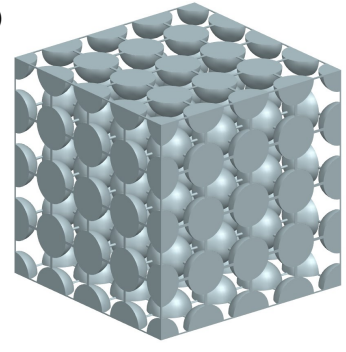

b)

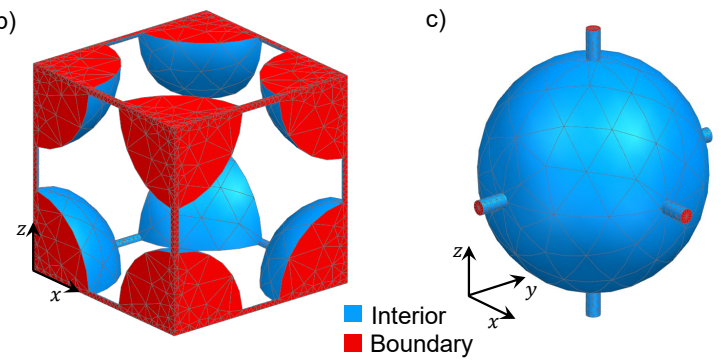

d)

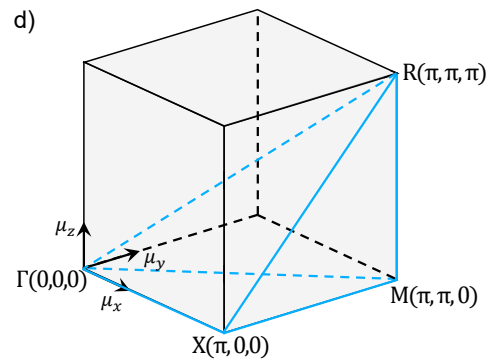

Fig. 1: a) periodic structure under investigation, b) lattice-based UC, c) shifted UC, d) IBC in reciprocal wave space

\section{Unit cell description}

This work aims to investigate the impact of the UC choice on the efficiency of the (G)BMS technique for 3D periodic structures. This is done by a numerical case study in which two UCs, representing the same 3D periodic structure, are compared in terms of the FOM and ROM dispersion curve calculation. More specific, the 3D phononic crystal proposed by Lucklum et al. [5] is employed in this work (Fig. 1(a)). This periodic structure consists of solid spheres connected by thin elastic circular rods in a simple cubic Bravais lattice. Figure 1(b) displays the UC used in the reference paper and will be referred to as the lattice-based UC in what follows. For this translational periodic structure, the choice of UC is not unique. As seen in Fig. 1(b), the lattice-based UC contains a lot of boundary DOFs which can be less interesting to apply the BMS technique, cf. Section 2. By shifting the UC over all three axes with half the lattice constant, an equivalent representation is obtained with the same amount of symmetry, visualized in Fig. 1(c). This UC contains very few boundary DOFs and is further denoted as the shifted UC. Both UCs have a lattice constant of $10 \mathrm{~mm}$, while the diameter of the spheres equals $7.9 \mathrm{~mm}$ and the circular beam diameter is $0.5 \mathrm{~mm}$. The material constants are taken from [5]: Young's modulus $E=3.2 \mathrm{GPa}$, mass density $\rho=1190 \mathrm{~kg} / \mathrm{m}^{3}$ and Poisson ratio $\nu=0.35$.

For the analysis, the undamped UCs are discretized using a FE approach in Siemens Simcenter 3D. The meshes of both representations are equal, i.e. the same mesh applying tetrahedral elements is used for one eight of the UCs and afterwards symmetry is exploited to construct the total mesh. Consequently, both meshes contain an equal amount of 4040 elements. Due to the different UC choice, a different number of nodes apply for both meshes, leading to a different amount of DOFs. Tab. 1 gives an overview. The system matrices are extracted from the commercial FE software and all further computations, i.e. the reduction and dispersion curve calculations, have been implemented in Matlab R2019b and are performed on a laptop with $32 \mathrm{~GB}$ RAM and a $2.6 \mathrm{GHz}$ Intel Core $17-9540 \mathrm{H}$ processor. The considered frequency range of interest is 0 to $10 \mathrm{kHz}$. By exploiting the symmetry of the UC, the dispersion curves are calculated along the IBC shown in Fig. 1(d) [22]. A resolution of $0.02 \pi$ is chosen between consecutive $\left(\mu_{x}, \mu_{y}, \mu_{z}\right)$-sets leading to 430 evaluation points.
Table 1: Number of nodes and DOFs in the FOM UCs

\begin{tabular}{c|c|ccc}
\hline & & \multicolumn{3}{|c}{ DOFs } \\
UC & Nodes & Total & Interior & Boundary \\
\hline Lattice-based & 9304 & 27912 & 10476 & 17436 \\
Shifted & 6269 & 18807 & 18141 & 666 \\
\hline
\end{tabular}

\section{Results}

In this section, the impact of the UC choice on the dispersion curve calculations is analyzed. First, a comparison is made between the dispersion curve calculation of both FOMs. Next, this section elaborates on the impact of the UC choice on the efficiency of the (G)BMS technique.

\subsection{Comparison of FOMs}

In this first comparison, the dispersion curve calculations using both FOM UCs are assessed. Although the lattice-based free UC contains more DOFs than the shifted UC, application of the Bloch-Floquet BCs leads to $18474 \mathrm{DOFs}$ for the resulting dispersion eigenvalue problem in both cases. Figure 2 shows the dispersion curves in the frequency range of interest, i.e. the first six modes, for both UCs together with the displacement of the UCs at points $\square, \bigcirc$ and $\diamond$ of the dispersion curves. Since both UC models represent the same infinite periodic structure, the corresponding dispersion curves and modeshapes are as good as identical (relative error of $10^{-7}$ ). The mode on point $\diamond$ represents an axial mode, while the ones on points $\square, \bigcirc$ are higher order bending modes. By inspecting the visualized mode shapes, it is evident that the main dynamics of the UCs are comprised in the connecting legs. In the case of the lattice-based UC, this relates to a large extent to its boundary DOFs, whereas for the shifted UC, this mainly relates to its interior DOFs.

Although both UC choices contain the same number of DOFs after applying the Bloch-Floquet BCs and lead to visually identical dispersion curves, the dispersion curve calculation takes longer for the lattice-based UC (Tab. 2). Both the execution of the Bloch-Floquet BCs and the eigenvalue calculation impact this difference in calculation time. The ap- 


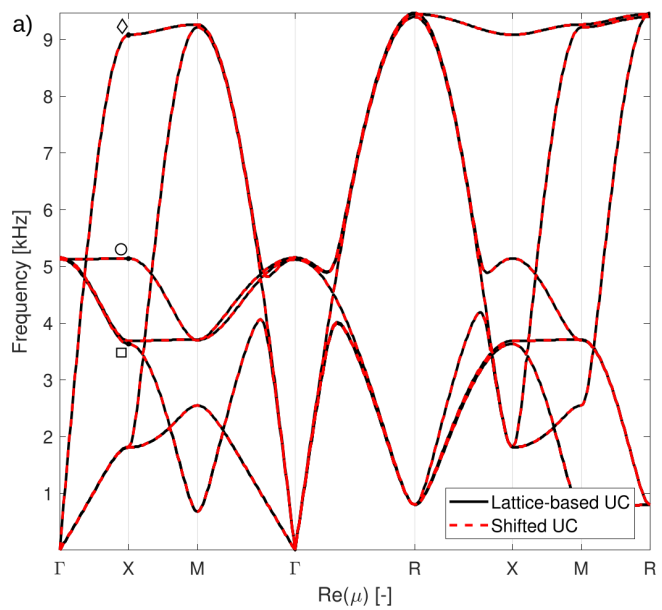

b)
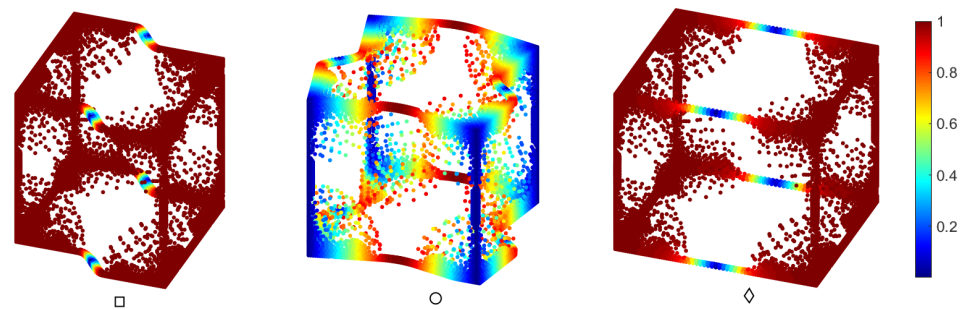

c)
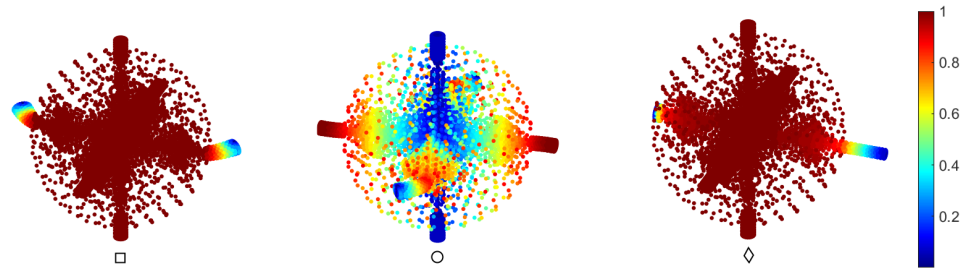

Fig. 2: a) Dispersion curve calculated using lattice-based and shifted UC. b) Left to right: modeshapes on points $\square$, $\bigcirc$ and $\diamond$ of the dispersion curve for the lattice-based UC. c) Left to right: modeshapes on points $\square, \bigcirc$ and $\diamond$ of the dispersion curve for the shifted UC. Coloring of the modeshapes represents the normalized magnitude of the displacement.

Table 2: Calculation time of FOM dispersion curves

\begin{tabular}{c|ccc}
\hline UC & Total & Bloch-Floquet BCs & Eigenvalues \\
\hline Lattice-based & $953 \mathrm{~s}$ & $112.5 \mathrm{~s}$ & $832.0 \mathrm{~s}$ \\
Shifted & $809 \mathrm{~s}$ & $38.8 \mathrm{~s}$ & $762.1 \mathrm{~s}$ \\
\hline
\end{tabular}

plication of the Bloch-Floquet BCs requires a higher computational effort for the lattice-based $\mathrm{UC}$, since the matrixmultiplication in Eq. (3) is performed on larger matrices, i.e. both the system matrices $\mathbf{M}$ and $\mathbf{K}$ as the periodicity matrix $\boldsymbol{\Lambda}$ are larger for the lattice-based UC relative to the shifted UC. The system matrices after application of the BlochFloquet BCs are visualized in Fig. 3. Although the matrices of the lattice-based UC contain slightly more zero-elements than the matrices of the shifted UC, the structure of the system matrices of the shifted UC behaves more as the blockdiagonal matrix structure encountered in standard FE eigenvalue problems [23]. This difference in structure impacts the efficiency to solve the general eigenvalue problem [24].

\subsection{Comparison of ROMs}

In previous section it is shown that even for the FOM, a smart choice of UC can lead to a faster calculation of the dispersion curves based on an equally accurate mesh. However, the calculation time remains cumbersome for both UCs. Therefore, reduction techniques are exploited to minimize the computation time. In this section, it is investigated whether the shifted UC is also preferred when the (G)BMS techniques are applied and what the impact of the $\mathrm{UC}$ choice is on the efficiency of these techniques. The number of modes $n_{I}$ and $n_{A}$ used to construct the ROMs for both UCs are given in Tab. 3. The application of (G)BMS to both UC models are compared by analyzing the resulting dispersion curves, the accuracy of the ROMs and computa-
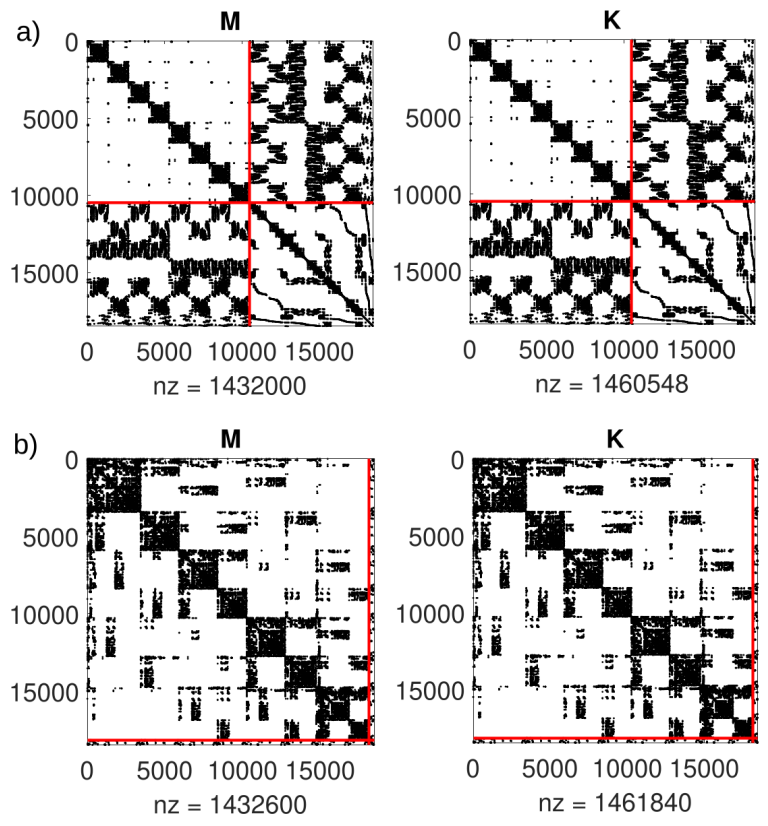

Fig. 3: Mass (M) and stiffness (K) matrix for both latticebased UC (a) and shifted UC (b) after applying BlochFloquet BCs at point $X$ of the dispersion curve. The red lines distinguish the part corresponding to the interior (upper left) and boundary (lower right) DOFs. The number of non-zero elements is indicated with $n z$.

tion times, see Figs. 4 and 5. The accuracy of the (G)BMS techniques are visualized using the relative frequency error:

$$
\epsilon_{r e l}=\left|\frac{\omega_{R O M}-\omega_{F O M}}{\omega_{F O M}}\right| .
$$

To avoid singularities at the point $\Gamma$ due to $0 \mathrm{~Hz}$ frequency solutions, these results are omitted from the $\epsilon_{r e l}$ calculation. A good accuracy can be obtained for both UCs, but the effi- 
ciency of the MOR techniques differs. Note that the computation time required to determine the dispersion curves with the ROMs contains the calculation of the modal basis, the modal reduction step and solving the dispersion eigenvalue problem. Using the (G)BMS technique with the shifted UC leads to a significant reduction in computation time. On the contrary, for the lattice-based UC, application of the BMS and GBMS technique leads to computation times which exceed the FOM based calculations. In what follows, these outcomes are discussed in more detail.

Table 3: Choice of the number of modes taken into account to construct the ROMs.

\begin{tabular}{c|cc}
\hline $\mathrm{UC}$ & $n_{I}$ & $n_{A}$ \\
\hline \begin{tabular}{c|c} 
Lattice-based \\
Shifted
\end{tabular} & 1 & 1207 \\
\hline
\end{tabular}

\subsubsection{BMS}

With $n_{I}=1$ taken into account in the BMS basis for the lattice-based UC, a ROM of 17437DOFs is obtained, which is further reduced to 7999DOFs after applying the Bloch-Floquet BCs. Although this ROM represents the dispersion curves with a good accuracy of order $10^{-4}$ or lower, it is not profitable to perform the reduction time-wise (cf. Fig. 4). The large increase in computation time has different causes. (i) Although the interior DOFs are reduced from 10476 to 1 , the number of boundary DOFs is unchanged, namely 17436 DOFs. This leads to a large dense matrix $\boldsymbol{\Psi}_{I A}$ containing the static constraint modes and consequently, the reduction base $\mathbf{B}$ remains of large dimension. Due to the large dimensions of both the system matrices and the reduction base, the required projections to construct the BMS ROM, i.e. the matrix-multiplications of Eq. (4), are computationally demanding. They require $196.2 \mathrm{~s}$ of the total required interior reduction step of $212.3 \mathrm{~s}$. (ii) Next, the sparse FOM system matrices $\mathbf{M}$ and $\mathbf{K}$ become fully dense after applying the MOR due to the static constraint modes, cf. Section 2.2. This dense character causes the computation time of the dispersion curve calculation to heavily increase. Due to the remaining relatively high dimension of the system matrices together with the dense character, both the required projections to apply the Bloch-Floquet BCs (Eq. (3)) and the eigenvalue problem of Eq. (2) take much more time than in the FOM case (Fig. 4).

Using the shifted UC instead, these problems are not encountered (Fig. 5). By applying the same frequency-based truncation criterion as done for the lattice-based UC, an $n_{I}=6$ is obtained in this case. With this choice, a ROM of $672 \mathrm{DOF}$ is constructed, further reduced to 339DOFs after applying the Bloch-Floquet BCs. Although a slightly higher amount of interior modes is calculated, it is based on a smaller UC model with significantly lower number of boundary DOFs (Tab. 1). In addition, the lower number of boundary DOFs leads to a significantly smaller ROM for this UC. Despite also being dense, the significantly smaller ROM matrices enable considerably faster dispersion curve calculations than for the lattice-based UC ROM as well as the FOM (Fig. 5). It is noted that the resulting accuracy in this case shows a smaller relative error as compared to the latticebased UC although the same frequency-dependent truncation criterion is used to select $n_{I}$.

\subsubsection{GBMS}

For the GBMS technique, the problems of the BMS propagate and similar conclusions can be drawn. Although the GBMS builds further upon the BMS, not the entire calculation of the BMS reduced matrices, cf. Eq. (4), is a prerequisite. The computationally demanding projections are partially executed to obtain the sub-matrices $\mathcal{M}_{A A}$ and $\mathcal{K}_{A A}$ while constructing the GBMS ROMs. For this reason, the reported interior modal reduction time is smaller in the GBMS case than in the BMS case (Figs. 4 and 5). The difference is especially evident for the lattice-based UC because in this case the matrix-multiplication dominates the BMS interior reduction step, as previously mentioned. Since in Section 4.1 it was found that the main dynamics for the lattice-based UC are comprised in the UC boundary for the considered frequency range, a high number of boundary modes is required when applying the GBMS technique. By gradually increasing $n_{A}$, an $n_{A}=1207$ was eventually found to lead to an acceptable accuracy, while still enabling a reduction in the UC model. As a consequence, the boundary modal reduction step for the lattice-based UC is computationally demanding (Fig. 4) since 1207 modes need to be calculated from the large fully dense matrices $\mathcal{M}_{A A}$ and $\mathcal{K}_{A A}$. For this case, the built-in function eig of Matlab was found to be more suitable than eigs due to the high number of required modes. For this lattice-based UC, the eventual resulting GBMS ROM size equals 14521, further reduced to 6541 after applying the Bloch-Floquet BCs. Although the computation cost of the dispersion curve calculation is reduced with respect to the BMS ROM, the total computation time still exceeds that of the FOM (Fig. 4). Similarly to the BMS, the explanation lies in the fully dense and still relatively large system matrices.

Applying the GBMS to the shifted UC, a considerable reduction in computation time is achieved relative to the FOM based and the BMS based dispersion curve calculation. In Section 4.1, it was learned that most of the dynamics for the shifted UC are comprised in the UC interior part for the considered frequency range. Therefore, only a small number of boundary modes, i.e. $n_{A}=30$, are required for the GBMS basis to obtain a reduction of the UC model while preserving a good accuracy. This smaller number has several advantages. (i) Firstly, less modes need to be calculated from the dense system matrices $\mathcal{M}_{A A}$ and $\mathcal{K}_{A A}$. Moreover, these matrices have a smaller dimension relative to the ones of the lattice-based UC due to the small number of bound- 
(a)
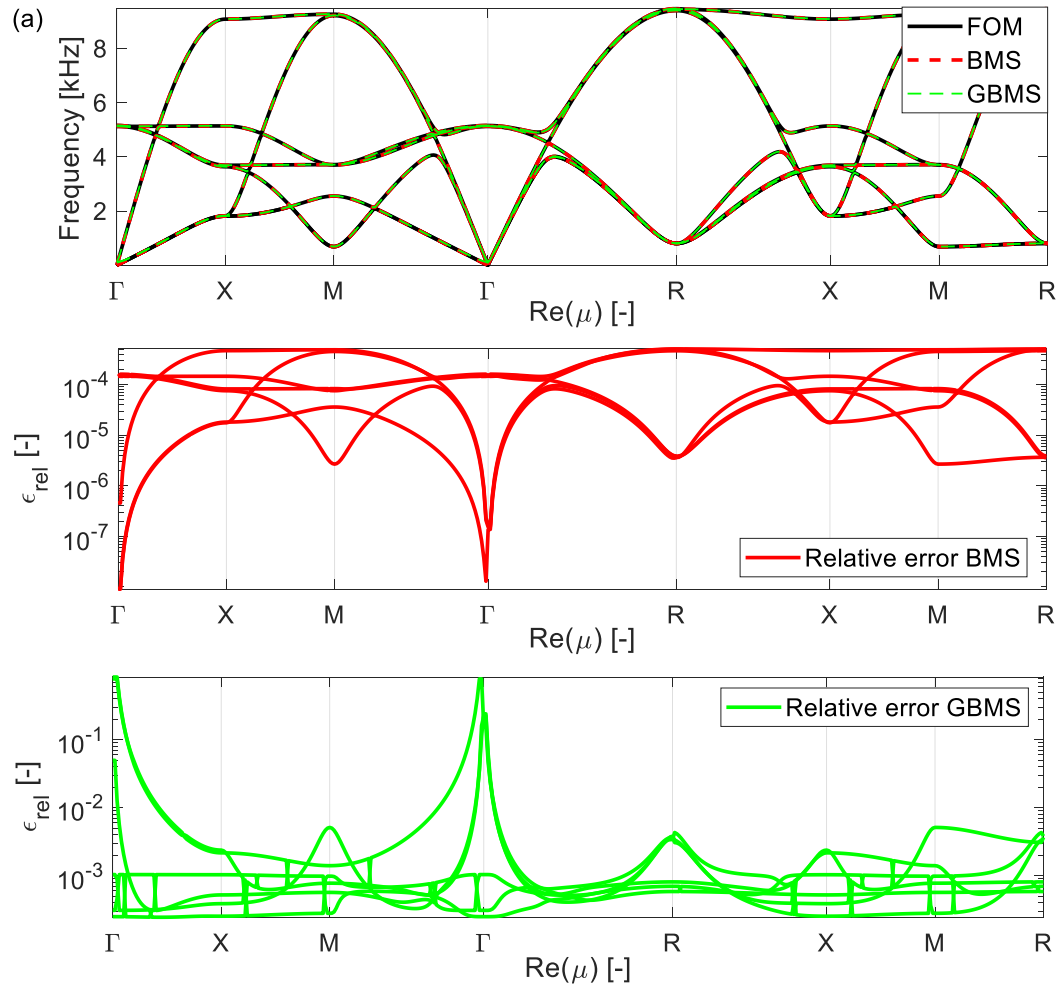

(b)

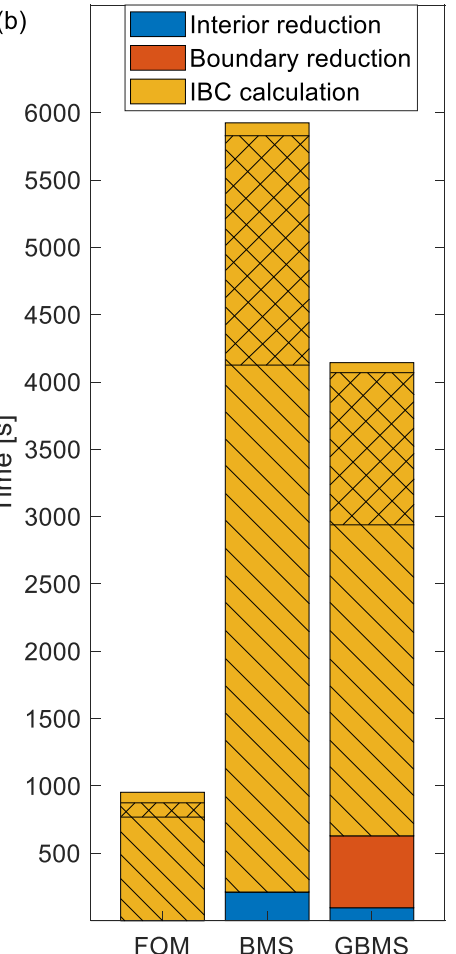

Fig. 4: a) Dispersion curves of lattice-based UC with underneath relative errors. b) Computation times of ROM set-up and dispersion curve calculation for lattice-based UC. The IBC calculation time is further divided: denotes the time to apply the Bloch-Floquet BCs (Eq. (3)), 제 denotes the time to compute the eigenvalue problem (Eq. (2)).

(a)
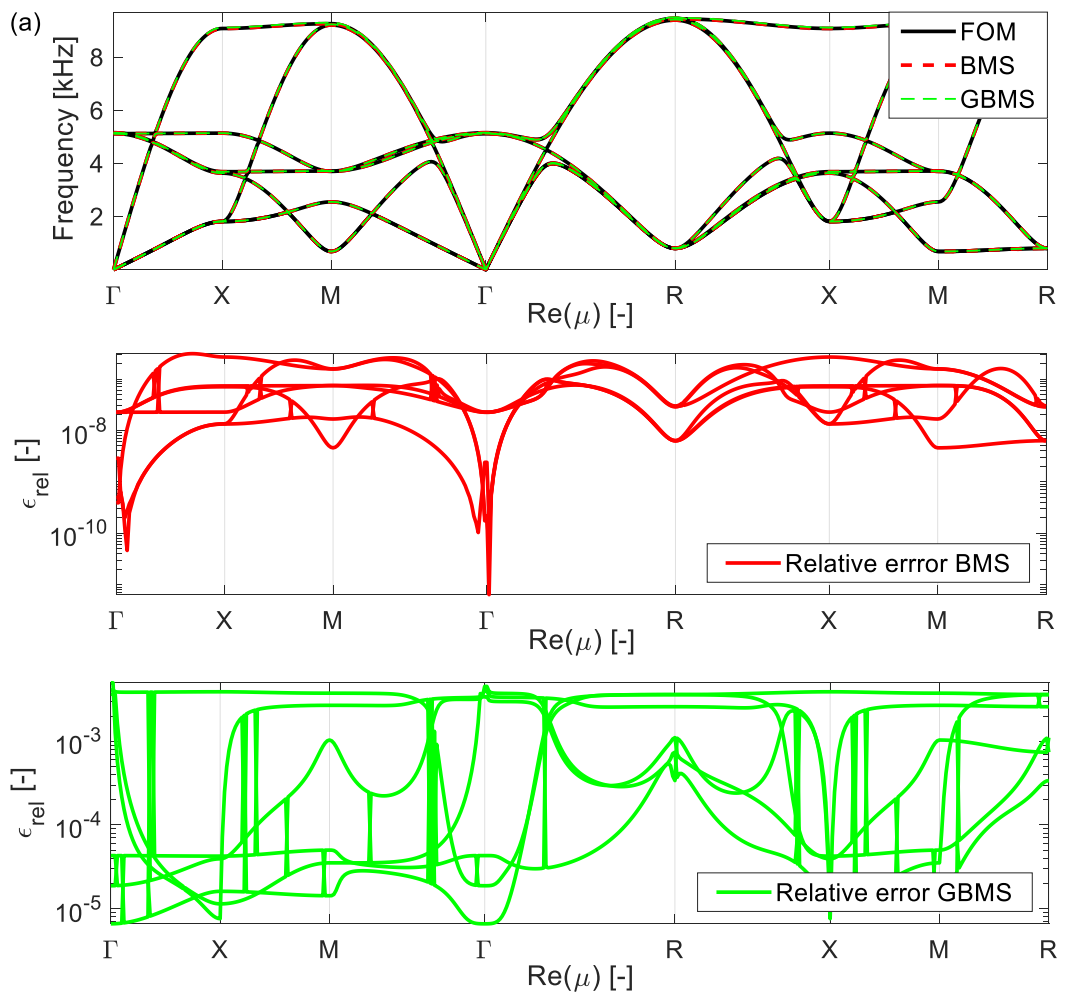

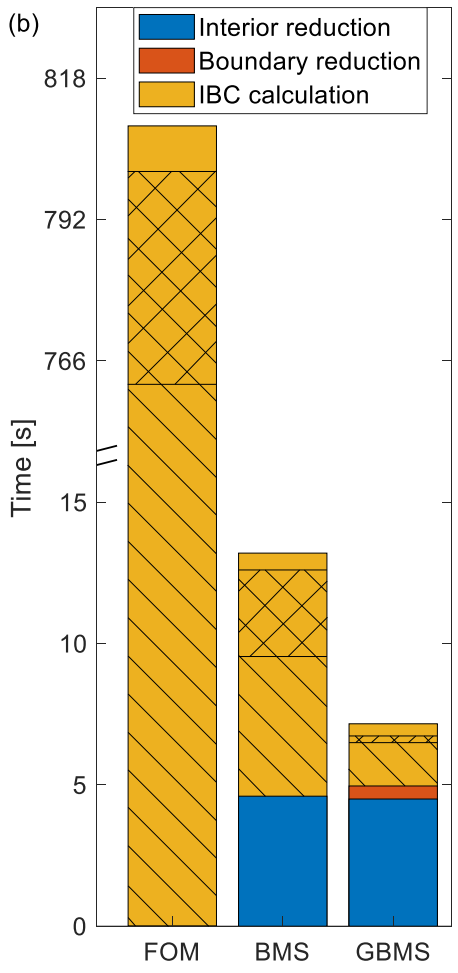

Fig. 5: a) Dispersion curves of shifted UC with underneath relative errors. b) Computation times of ROM set-up and dispersion curve calculation for shifted UC. A break in the time axis is present. The IBC calculation time is further divided: denotes the time to apply the Bloch-Floquet BCs (Eq. (3)), N denotes the time to compute the eigenvalue problem (Eq. (2)). 
ary DOFs. (ii) Secondly, a very small ROM is obtained. With $n_{A}=30$, the GBMS technique leads to a reduced system of only $128 \mathrm{DOFs}$ and this is further reduced to $67 \mathrm{DOF}$ after the Bloch-Floquet BCs. Although the same characteristics as for the lattice-based UC hold, i.e. demanding eigenvalue problems and dense matrices, the calculation time is strongly reduced due to the small amount of remaining DOFs (Fig. 5).

\section{Conclusions}

In this paper, the $(\mathrm{G}) \mathrm{BMS}$ technique was applied to a finite element UC model of a 3D periodic phononic crystal. The impact of the UC choice on the efficiency of the reduction techniques is investigated with a numerical case study. Two UCs representing the same 3D periodic structure, but with another configuration, are compared. The UCs mainly differ in the ratio of interior to boundary DOFs and consequently whether or not the dynamic information is included in the interior or boundary part. The dispersion curves calculated using both full order UC models are identical. However, the difference in configuration leads to a different computation time required. The UC with less boundary DOFs is favorable, however, both dispersion curve calculations remain cumbersome to compute. When applying the (G)BMS techniques, significant differences in computational efficiency occur between different UC choices. In particular, the UC with a high amount of boundary DOFs negatively impacts the efficiency of the reduction techniques as the computation is considerable worse than the UC with less boundary DOFs and the FOM. The differences were explained by the size and structure of the reduction bases and resulting ROMs. In conclusion, it is advisable to make a thoughtful decision for the UC choice to facilitate a fast FOM and ROM dispersion curve calculation. In this work, it was found to be favorable to diminish the number of boundary DOFs of the UC and contain the main dynamics in the interior part.

\section{Acknowledgements}

The research of V. Cool (fellowship no. 11G4421N) and L. Van Belle (fellowship no. 1271621N) is funded by a grant from the Research Foundation - Flanders (FWO). The Research Fund KU Leuven is gratefully acknowledged for their support. The work leading to this publication has been partially funded by the project MOR4MDesign, which fits in the MacroModelMat (M3) research program, coordinated by Siemens (Siemens Digital Industries Software, Belgium) and funded by SIM (Strategic Initiative Materials in Flanders) and VLAIO (Flanders Innovation \& Entrepreneurship Agency).

\section{References}

[1] Hussein, M., Leamy, M., and Ruzzene, M., 2014. "Dynamics of phononic materials and structures: Historical origins, recent progress, and future outlook". Applied Mechanics Reviews, 66(4).
[2] Xiao, Y., Wen, J., Wang, G., and Wen, X., 2013. "Theoretical and experimental study of locally resonant and Bragg band gaps in flexural beams carrying periodic arrays of beam-like resonators". Journal of Vibration and Acoustics, 135(4).

[3] Claeys, C., Deckers, E., Pluymers, B., and Desmet, W., 2016. "A lightweight vibro-acoustic metamaterial demonstrator: Numerical and experimental investigation”. Mechanical systems and signal processing, 70, pp. 853-880.

[4] D’Alessandro, L., Belloni, E., Ardito, R., Corigliano, A., and Braghin, F., 2016. "Modeling and experimental verification of an ultra-wide bandgap in 3D phononic crystal". Applied Physics Letters, 109(22), p. 221907.

[5] Lucklum, F., and Vellekoop, M., 2018. "Bandgap engineering of three-dimensional phononic crystals in a simple cubic lattice". Applied Physics Letters, 113(20).

[6] Krattiger, D., and Hussein, M., 2018. "Generalized Bloch mode synthesis for accelerated calculation of elastic band structures". Journal of Computational Physics, 357, pp. 183-205.

[7] Bloch, F., 1929. "Über die quantenmechanik der elektronen in kristallgittern". Zeitschrift für physik, 52(7), pp. 555-600.

[8] Mace, B., and Manconi, E., 2008. "Modelling wave propagation in two-dimensional structures using finite element analysis". Journal of Sound and Vibration, 318(4-5), pp. 884-902.

[9] Benner, P., Schilders, W., Grivet-Talocia, S., et al., 2020. Model Order Reduction: Volume 3 Applications. De Gruyter.

[10] van de Walle, A., Naets, F., Deckers, E., and Desmet, W., 2017. "Stability-preserving model order reduction for time-domain simulation of vibro-acoustic FE models". International Journal for Numerical Methods in Engineering, 109(6), pp. 889-912.

[11] Chen, Y., Zhang, B., Zhang, N., and Zheng, M., 2015. "A condensation method for the dynamic analysis of vertical vehicle-track interaction considering vehicle flexibility". Journal of Vibration and Acoustics, 137(4).

[12] De Gregoriis, D., Naets, F., Kindt, P., and Desmet, W., 2019. "Application of a priori hyper-reduction to the nonlinear dynamic finite element simulation of a rolling car tire". Journal of Computational and Nonlinear Dynamics, 14(11).

[13] An, X., Lai, C., Fan, H., and Zhang, C., 2020. "3D acoustic metamaterial-based mechanical metalattice structures for low-frequency and broadband vibration attenuation". International Journal of Solids and Structures, 191, pp. 293-306.

[14] Hussein, M., 2009. "Reduced Bloch mode expansion for periodic media band structure calculations". Proceedings of the Royal Society A: Mathematical, Physical and Engineering Sciences, 465(2109), pp. 28252848.

[15] Boukadia, R., Droz, C., Ichchou, M., and Desmet, W., 2018. "A Bloch wave reduction scheme for ultrafast band diagram and dynamic response computation in 
periodic structures". Finite Elements in Analysis and Design, 148, pp. 1-12.

[16] Droz, C., Zhou, C., Ichchou, M., and Lainé, J.-P., 2016. "A hybrid wave-mode formulation for the vibroacoustic analysis of 2D periodic structures". Journal of Sound and Vibration, 363, pp. 285-302.

[17] Krattiger, D., and Hussein, M., 2014. "Bloch mode synthesis: ultrafast methodology for elastic bandstructure calculations". Physical Review E, 90(6).

[18] Krattiger, D., Wu, L., Zacharczuk, M., et al., 2019. "Interface reduction for Hurty/Craig-Bampton substructured models: Review and improvements". Mechanical Systems and Signal Processing, 114, pp. 579-603.

[19] Van Belle, L., de Melo Filho, N., et al., 2020. "Fast metamaterial design optimization using reduced order unit cell modeling". Proceedings of ISMA 2020.

[20] Kittel, C., 2010. Introduction to solid state physics. Wiley New York.

[21] Herrmann, J., Maess, M., and Gaul, L., 2010. "Substructuring including interface reduction for the efficient vibro-acoustic simulation of fluid-filled piping systems". Mechanical systems and signal processing, 24(1), pp. 153-163.

[22] Setyawan, W., and Curtarolo, S., 2010. "Highthroughput electronic band structure calculations: Challenges and tools". Computational materials science, 49(2), pp. 299-312.

[23] Zienkiewicz, O., and Taylor, R., 1994. The finite element method; volume 1: basic formulation and linear problems.

[24] Golub, G., and Van der Vorst, H., 2000. "Eigenvalue computation in the 20th century". Journal of Computational and Applied Mathematics, 123(1-2), pp. 35-65. 